\title{
FINITE ASPHERICAL COMPLEXES WITH INFINITELY-GENERATED GROUPS OF SELF-HOMOTOPY-EQUIVALENCES
}

\author{
DARRYL MCCULLOUGH
}

\begin{abstract}
A finite aspherical complex $X$ is constructed whose group of homotopy classes of basepoint-preserving self-homotopy-equivalences is infinitely generated.
\end{abstract}

0. Introduction. Let $E X$ denote the $H$-space of basepoint-preserving homotopy equivalences from $X$ to $X$. The set $\pi_{0}(E X)$ becomes a group with multiplication induced by composition of representatives; it is denoted $\mathcal{E}(X)$ and called, by abuse of language, the group of homotopy equivalences of $X$.

It was proved independently by $\mathrm{D}$. Sullivan [Su] and C. Wilkerson [W] that $\mathcal{E}(X)$ is finitely presented when $X$ is a simply-connected finite complex. In contrast, Frank and Kahn [F-K] showed that $\mathcal{E}\left(S^{1} \vee S^{p} \vee S^{2 p-1}\right)$ is infinitely generated for $p \geqslant 2$. They also suggested the following alternative method for producing examples. Start with Lewin's [L] example of a finitely-presented group $G$ with $\operatorname{Aut}(G)$ infinitely generated. It is well known (see e.g. [S, p. 427]) that if $X$ is a $K(\pi, 1)$-complex then $\mathcal{E}(X) \cong \operatorname{Aut}(\pi)$, and therefore any $K(G, 1)$-complex would have an infinitely-generated group of homotopy equivalences. In this note, I will construct a finite $K(G, 1)$-complex $X$, note some of its salient features, and raise some questions.

1. Construction of $X$. Let $G_{Y}$ be the group with presentation

$$
P_{Y}=\left\langle a, b, x:[a, b]=1, x^{-1} a x=a^{2}, x^{-1} b x=b^{2}\right\rangle
$$

and let $G_{M}$ be the group with presentation

$$
P_{M}=\left\langle a, b, y:[a, b]=1, y^{-1} a y=a, y^{-1} b y=b a\right\rangle .
$$

We will construct a $K\left(G_{Y}, 1\right)$-complex $Y$ and a $K\left(G_{M}, 1\right)$-complex $M$.

Let exp: $\mathrm{C} \rightarrow \mathrm{C}$ be the exponential map. Let $T=S^{1} \times S^{1} \subset C^{2}$ and let $p_{a}, p_{b}$ : $I \rightarrow T$ be the loops $p_{a}(t)=(\exp (2 \pi i t), 1)$ and $p_{b}(t)=(1, \exp (2 \pi i t))$. Then $a=\left\langle p_{a}\right\rangle$ and $b=\left\langle p_{b}\right\rangle$ generate $\pi_{1}(T,(1,1)) \cong \mathbf{Z} \oplus \mathbf{Z}$. If $f: T \rightarrow T$ is the 4-fold covering map defined by

$$
f\left(\exp \left(2 \pi i t_{1}\right), \exp \left(2 \pi i t_{2}\right)\right)=\left(\exp \left(4 \pi i t_{1}\right), \exp \left(4 \pi i t_{2}\right)\right)
$$

Received by the editors September 7, 1979.

AMS (MOS) subject classifications (1970). Primary 55D10, 55E05; Secondary 20E40, 20F55, 55D20, $57 \mathrm{~B} 10$.

Key words and phrases. Self-homotopy-equivalence, aspherical complex, Higman-Neumann-Neumann group, automorphism group, duality group. 
then $f_{\#}(a)=a^{2}$ and $f_{\#}(b)=b^{2}$. Let $Y$ be the mapping torus

$$
Y=T \times I /(w, 0) \sim(f(w), 1) .
$$

Let $p_{x}: I \rightarrow Y$ be the loop $p_{x}(t)=[(1,1), t]$, and let $x=\left\langle p_{x}\right\rangle \in \pi_{1}(Y,[(1,1), 0])$. The fundamental group of $Y$ is a Higman-Neumann-Neumann (HNN) group with presentation $P_{Y}$. To see directly that $Y$ is aspherical, consider its infinite cyclic covering space $\tilde{Y}$ corresponding to the commutator subgroup (= the normal closure of $a$ and $b$ ) of $G_{Y}$. It consists of countably many 3-dimensional aspherical pieces attached along aspherical subsets, and we use Theorem 5 of [Wh] and direct limits to see that $\tilde{Y}$ is aspherical. Therefore $Y$ is a 3-dimensional finite $K\left(G_{Y}, 1\right)$ complex.

Let $h: T \rightarrow T$ be the homeomorphism given by

$$
h\left(\exp \left(2 \pi i t_{1}\right), \exp \left(2 \pi i t_{2}\right)\right)=\left(\exp \left(2 \pi i\left(t_{1}+t_{2}\right)\right), \exp \left(2 \pi i t_{2}\right)\right) .
$$

Then $h_{\#}(a)=a$ and $h_{\#}(b)=b a$. Form the closed aspherical 3-manifold

$$
M=T \times I /(w, 0) \sim(h(w), 1) .
$$

It is a bundle over the circle with torus fiber. Let $y \in \pi_{1}(M,[(1,1), 0])$ be $\left\langle p_{y}\right\rangle$ where $p_{y}(t)=[(1,1), t]$. Then $\pi_{1}(M)$ is an HNN group with presentation $P_{M}$, so $M$ is a 3-dimensional $K\left(G_{M}, 1\right)$-manifold.

The complex $X$ can be writen as a mapping torus in two ways.

$$
X=Y \times I /[[w, r], 0] \sim[[h(w), r], 1]
$$

or

$$
X=M \times I /[[v, s], 0] \sim[[f(v), s], 1] .
$$

$X$ is 4-dimensional and is aspherical by the same reasoning as we used for $Y$. The fundamental group of $X$ is an HNN group of $G$ with presentation

$$
\begin{aligned}
P_{G}=\left\langle a, b, x, y:[a, b]=1,[x, y]=1, x^{-1} a x\right. & =a^{2}, \\
x^{-1} b x & \left.=b^{2}, y^{-1} a y=a, y^{-1} b y=b a\right\rangle
\end{aligned}
$$

so $X$ is a finite $K(G, 1)$-complex.

2. Properties of $X$. (1) It was shown by Lewin $[L]$ that $\operatorname{Aut}(G)$ is infinitely generated. Therefore $\mathcal{E}(X) \cong \operatorname{Aut}(G)$ is infinitely generated.

(2) By $9.16(\mathrm{~b})$ of [B], $G$ is a duality group of dimension 4 over $Z$, therefore $X$ is a duality complex. But $G$ is not a Poincaré duality group, since $H^{4}(G ; \mathrm{ZG}) \cong$ $H_{\text {finite }}^{4}(\tilde{X} ; Z)$ is an infinitely-generated group, so $X$ is not a Poincaré complex.

(3) By making a few small changes we can produce infinitely many similar examples. There are groups $G_{m}$ for all integers $m>1$, with $G_{1}=G$, such that

(a) $\operatorname{Aut}\left(G_{m}\right)$ is infinitely generated.

(b) $G_{m} \cong G_{n}$ if and only if $m=n \cdot 2^{k}$ for some $k \in \mathbf{Z}$.

Presentations for the $G_{m}$ and a proof of (b) are given in an addendum to this note. The proof of (a) is a slight modification of the argument of Lewin [L]. For each $m>1$, by replacing the map $h=h_{1}$ of $\S 1$ by $h_{m}: T \rightarrow T$ where

$$
h_{m}\left(\exp \left(2 \pi i t_{1}\right), \exp \left(2 \pi i t_{2}\right)\right)=\left(\exp \left(2 \pi i\left(t_{1}+m \cdot t_{2}\right)\right), \exp \left(2 \pi i t_{2}\right)\right) \text {, }
$$

we produce a closed aspherical 3-manifold $M_{m}$ contained in a 4-dimensional 
aspherical complex $X_{m}$ with $\pi_{1}\left(X_{m}\right) \cong G_{m}$. By (b), infinitely many homotopy types appear among the $X_{m}$. The manifolds $M_{m}$ appear in a different context in [R-S].

3. Questions. (1) The Frank and Kahn examples and the $X_{m}$ are dissimilar examples of finite complexes $Z$ with $\mathcal{E}(Z)$ infinitely generated. Are there other types of examples? Is there a 2-dimensional example?

(2) Are there examples for which infinitely many generators of $\mathcal{E}(Z)$ can be represented by homeomorphisms? (There are closed 3-manifolds $M$ for which $\pi_{1}(E M)$ is infinitely generated and infinitely many generators can be represented by isotopies [M].)

(3) Can a closed $K(\pi, 1)$-manifold $N$ have $\mathcal{E}(N)$ infinitely generated?

(4) The groups $G_{m}$ are duality groups over $\mathbf{Z}$ but not Poincaré duality groups. Can a Poincaré duality group have an infinitely-generated automorphism group? An affirmative answer to (3) would provide an example of such a group [B, p. 170]. It is conceivable that (3) and (4) are equivalent.

(5) If $\pi$ is a finitely-presented group with $\operatorname{Aut}(\pi)$ infinitely generated, must $\pi$ contain infinitely-divisible elements?

Addendum: The groups $G_{m}$. For $m \geqslant 1$ let $G_{m}$ be the group with presentation

$$
\begin{aligned}
\left\langle a, b, x, y:[a, b]=1,[x, y]=1, x^{-1} a x\right. & =a^{2}, \\
x^{-1} b x & \left.=b^{2}, y^{-1} a y=a, y^{-1} b y=b a^{m}\right\rangle .
\end{aligned}
$$

Let $Z\left[\frac{1}{2}\right]$ be the additive group of dyadic rationals. We regard $G_{m}$ as a semidirect product

$$
1 \rightarrow \mathbf{Z}\left[\frac{1}{2}\right] \oplus \mathbf{Z}\left[\frac{1}{2}\right] \rightarrow G_{m} \rightarrow \mathbf{Z} \oplus \mathbf{Z} \rightarrow 1
$$

where $\mathbf{Z} \oplus \mathbf{Z}$ is the abelianization of $G_{m}$, generated by $x$ and $y$, and $\mathbf{Z}\left[\frac{1}{2}\right] \oplus \mathbf{Z}\left[\frac{1}{2}\right]$ is the commutator subgroup where $a$ and $b$ correspond to $(1,0)$ and $(0,1)$ respectively. The choice of basis $\{a, b\}$ determines an isomorphism $\operatorname{Aut}\left(\mathbf{Z}\left[\frac{1}{2}\right] \oplus \mathbf{Z}\left[\frac{1}{2}\right]\right) \simeq$ $G L_{2}\left(Z\left[\frac{1}{2}\right]\right)$ so that the action of $\mathbf{Z} \oplus \mathbf{Z}$ is given by $\phi_{m}: \mathbf{Z} \oplus \mathbf{Z} \rightarrow G L_{2}\left(Z\left[\frac{1}{2}\right]\right)$ defined by

$$
\phi_{m}(x)=\left(\begin{array}{ll}
2 & 0 \\
0 & 2
\end{array}\right) \text { and } \phi_{m}(y)=\left(\begin{array}{cc}
1 & m \\
0 & 1
\end{array}\right)
$$

Proposition. $G_{m} \cong G_{n}$ if and only if $m=n \cdot 2^{i}$ for some integer $i$.

Proof. Our proof uses elements from [L].

Suppose $f: G_{m} \cong G_{n}$. Since $Z\left[\frac{1}{2}\right] \oplus Z \mathbf{Z}\left[\frac{1}{2}\right]$ is the commutator subgroup we have a morphism of extensions

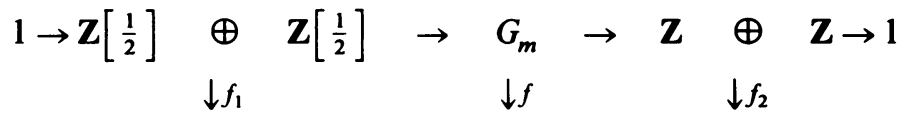

$$
\begin{aligned}
& 1 \rightarrow \mathbf{Z}\left[\frac{1}{2}\right] \quad \bigoplus \quad \mathbf{Z}\left[\frac{1}{2}\right] \quad \rightarrow \quad G_{n} \quad \rightarrow \quad Z \quad \bigoplus \quad Z \rightarrow 1
\end{aligned}
$$

We choose generators $A, B, X, Y$ for $G_{n}$ so that

$$
\phi_{n}(X)=\left(\begin{array}{ll}
2 & 0 \\
0 & 2
\end{array}\right) \text { and } \phi_{n}(Y)=\left(\begin{array}{ll}
1 & n \\
0 & 1
\end{array}\right)
$$


We regard $f_{1}$ as an element of $G L_{2}\left(Z\left[\frac{1}{2}\right]\right)$, written with respect to the bases $\{a, b\} \subset \mathbf{Z}\left[\frac{1}{2}\right] \oplus \mathbf{Z}\left[\frac{1}{2}\right] \subset G_{m}$ and $\{A, B\} \subset \mathbf{Z}\left[\frac{1}{2}\right] \oplus \mathbf{Z}\left[\frac{1}{2}\right] \subset G_{n}$. Commutativity of the diagram implies that

$$
f_{1} \phi_{m}(x) f_{1}^{-1}=\phi_{n}\left(f_{2}(x)\right),
$$

therefore $\left(\begin{array}{ll}2 & g \\ 0 & 2\end{array}\right)=\phi_{n}\left(f_{2}(x)\right)$ so $f_{2}(x)=X$. Since $f_{2}$ is an isomorphism, $f_{2}(y)=$ $X^{k} Y^{ \pm 1}$. Therefore

$$
f_{1} \phi_{m}(y) f_{1}^{-1}=\phi_{n}\left(X^{k} Y^{ \pm 1}\right)=\left(\begin{array}{cc}
2^{k} & 0 \\
0 & 2^{k}
\end{array}\right)\left(\begin{array}{cc}
1 & \pm n \\
0 & 1
\end{array}\right) .
$$

Since the determinant of $\phi_{m}(y)$ is 1 , we must have $k=0$, so

$$
f_{1}\left(\begin{array}{cc}
1 & m \\
0 & 1
\end{array}\right) f_{1}^{-1}=\left(\begin{array}{cc}
1 & \pm n \\
0 & 1
\end{array}\right)
$$

Writing $f_{1}=\left(\begin{array}{cc}p & q \\ r & s\end{array}\right)$, this equation becomes

$$
\frac{1}{p s-r q}\left(\begin{array}{cc}
p s-r q-m p r & m p^{2} \\
-m r^{2} & p s-q r+m p r
\end{array}\right)=\left(\begin{array}{cc}
1 & \pm n \\
0 & 1
\end{array}\right) \text {. }
$$

Therefore $r=0$ and we have

$$
\left(\begin{array}{cc}
p s & m p^{2} \\
0 & p s
\end{array}\right)=\left(\begin{array}{cc}
p s & \pm n p s \\
0 & p s
\end{array}\right)
$$

so $m p^{2}= \pm n p s$. Since $p s$ was a unit in the ring $\mathbf{Z}\left[\frac{1}{2}\right], p$ and $s$ are powers of 2 and we have $m=n \cdot 2^{i}$ for some $i$.

Conversely, if $m=n \cdot 2^{i}$ we define a homomorphism $f: G_{m} \rightarrow G_{n}$ on generators by $f(a)=X^{i} A X^{-i}, f(b)=B, f(x)=X, f(y)=Y$. The inverse of $f$ is $g$ defined by $g(A)=x^{-i} a x^{i}, g(B)=b, g(X)=x, g(Y)=y$, so $G_{m} \cong G_{n}$.

\section{BIBLIOGRAPHY}

[B] R. Bieri, Homological dimension of discrete groups, Queen Mary College Math. Notes, Univ. of London, 1976.

[F-K] D. Frank and D. W. Kahn, Finite complexes with infinitely-generated groups of self-equivalences, Topology 16 (1977), 189-192.

[L] J. Lewin, A finitely-presented group whose group of automorphisms is infinitely-generated, J. London Math. Soc. 42 (1967), 610-613.

[M] D. McCullough, Homotopy groups of the space of self-homotopy-equivalences, preprint.

[R-S] F. Raymond and L. Scott, The failure of Nielsen's theorem in higher dimensions, Arch. Math. 29 (1977), 643-654.

[S] E. Spanier, Algebraic topology, McGraw-Hill, New York, 1966.

[Su] D. Sullivan, Infinitesimal computations in topology, Inst. Hautes Études Sci. Publ. Math. 47 (1977), 269-331.

[W] C. W. Wilkerson, Applications of minimal simplicial groups, Topology 15 (1976), 111-130.

[Wh] J. H. C. Whitehead, On the asphericity of regions in a 3-sphere, Fund. Math. 32 (1939), 149-166.

Department of Mathematics, Universtty of OKLahoma, Norman, Okinhoma 73019 